\title{
Candida Albicans Peritoneal Infection In A Patient With Sclerosing Encapsulating Peritonitis Under Continuous Peritoneal Dialysis. Efficacy Of Intra-Abdominal Amphotericin B And Negative Pressure After Failure Of A Systemic Therapy.
}

\author{
B Oreste, B Valerio, S Loredana, S Giuseppina, G Vittoria, F Antonella, I \\ Benedetto, V Dario, P Giuseppe
}

\begin{abstract}
Citation
B Oreste, B Valerio, S Loredana, S Giuseppina, G Vittoria, F Antonella, I Benedetto, V Dario, P Giuseppe. Candida Albicans Peritoneal Infection In A Patient With Sclerosing Encapsulating Peritonitis Under Continuous Peritoneal Dialysis. Efficacy Of Intra-Abdominal Amphotericin B And Negative Pressure After Failure Of A Systemic Therapy.. The Internet Journal of Surgery. 2009 Volume 25 Number 1.
\end{abstract}

\begin{abstract}
Introduction: Fungal peritonitis related to peritoneal dialysis contributes significantly to morbidity and to a high level of mortality. Up to now, there have not been any studies clearly demonstrating which treatment regimen has the best outcome in this kind of patients.Case Report: A 33-year-old man, under continuous ambulatory peritoneal dialysis, was admitted to our department with suspected infective peritonitis. Culture results of peritoneal lavage showed growing of Enterococcus faecalis and colonies of Candida albicans. We decided to immediately explore the abdomen, to remove the peritoneal dialysis catheter and to administer intravenous fluconazole and amphotericin B. Despite 30 days of intravenous combined-antibiotic therapy, cultures were still positives for Candida albicans

. Peritoneal washing with amphotericin B and vacuum-assisted abdominal system were started and after 12 cycles we achieved the eradication of Candida. Discussion: Up to $80 \%$ of fungal peritonitis in patients on peritoneal dialysis are caused by candida. Catheter removal and prompt initiation of antifungal therapy are indicated; subsequent therapy is based upon culture results, sensitivity, and patient response. Vacuum-assisted wound closure has become the preferred treatment modality for many complex/chronic wounds and the topical negative pressure demonstrated a positive contribution also in managing severe peritonitis.Conclusions: Peritoneal sclerosis can negatively affect results of an intravenous antifungal therapy. Amphotericin $B$ intraperitoneal lavages associated with topical negative abdominal pressure can represent an effective option for common therapy-resistant peritonitis.
\end{abstract}

\section{BACKGROUND}

Fungal peritonitis related to peritoneal dialysis (PDCP) contributes significantly to morbidity and to a high level of mortality ${ }^{(1,2)}$. Up to now, there have not been any studies clearly demonstrating which treatment regimen has the best outcome in this kind of patients.

Peritoneum lavages, catheter removal and the prompt initiation of antifungal therapy are indicated; subsequent therapy is based upon culture results and patient response ${ }^{(3)}$. Some studies demonstrated that the peritoneal application of amphotericin B can improve the outcome of these patients in terms of technique survival, morbidity and mortality ${ }^{(4)}$. Vacuum-assisted wound closure (VAWC) has become the preferred treatment modality for many complex/chronic wounds and the topical negative pressure demonstrated a positive contribution in managing severe peritonitis. We report on a successfully managed case of a peritonealdialysis-related Candida peritonitis through the association of intravenous therapy, intra-peritoneal amphotericin B lavage and VAWC.

\section{CASE REPORT}

A 33-year-old man was admitted to our emergency 
department with shiver-preceded high-fever, epigastric pain, abdominal distension, rebound tenderness and vomiting. He had a history of autoimmune Berger disease and HCV infection that caused the failure of two consecutive kidney transplants (in 1996 and 2004). For this reason, he followed a programme of Continuous Ambulatory Peritoneal Dialysis (CAPD) for the last 28 months. An urgent dialysis was started and blood exams were requested. Lab results demonstrated leukocytosis and culture of peritoneal washing was performed, showing immediately a purulent raising and elevated leukocytes. An infective peritonitis was suspected and an empirical antibiotic therapy with intravenous administration of Ceftriaxone and Metronidazole was started. The culture results of peritoneal dialysis filtrate showed Enterococcus faecalis growing, as well as, colonies of Candida albicans. An urgent CT scan was performed (Figure 1b), showing small bowel distension and peritoneal thickening. No diverticulitis or appendicitis was suggested by imaging. In light of these findings, we decided to perform an emergency laparotomy (Figure 1a), to explore the abdomen and to immediately remove the peritoneal dialysis catheter. Parietal and visceral peritoneum appeared to be thickened and dotted of hyphae and fibrine branches. Small and large bowel were hard to explore precisely due the presence of peritoneal sclerosis, but no evidence of perforation or bowel ischemia were noticed. A biopsy of these findings was obtained. We made copious washes of the abdominal cavity; then we closed the laparotomy.

\section{Figure 1}

Figure 1a. Intraoperative findings showing peritoneal sclerosis with fibrine branches and Candida hyphes.

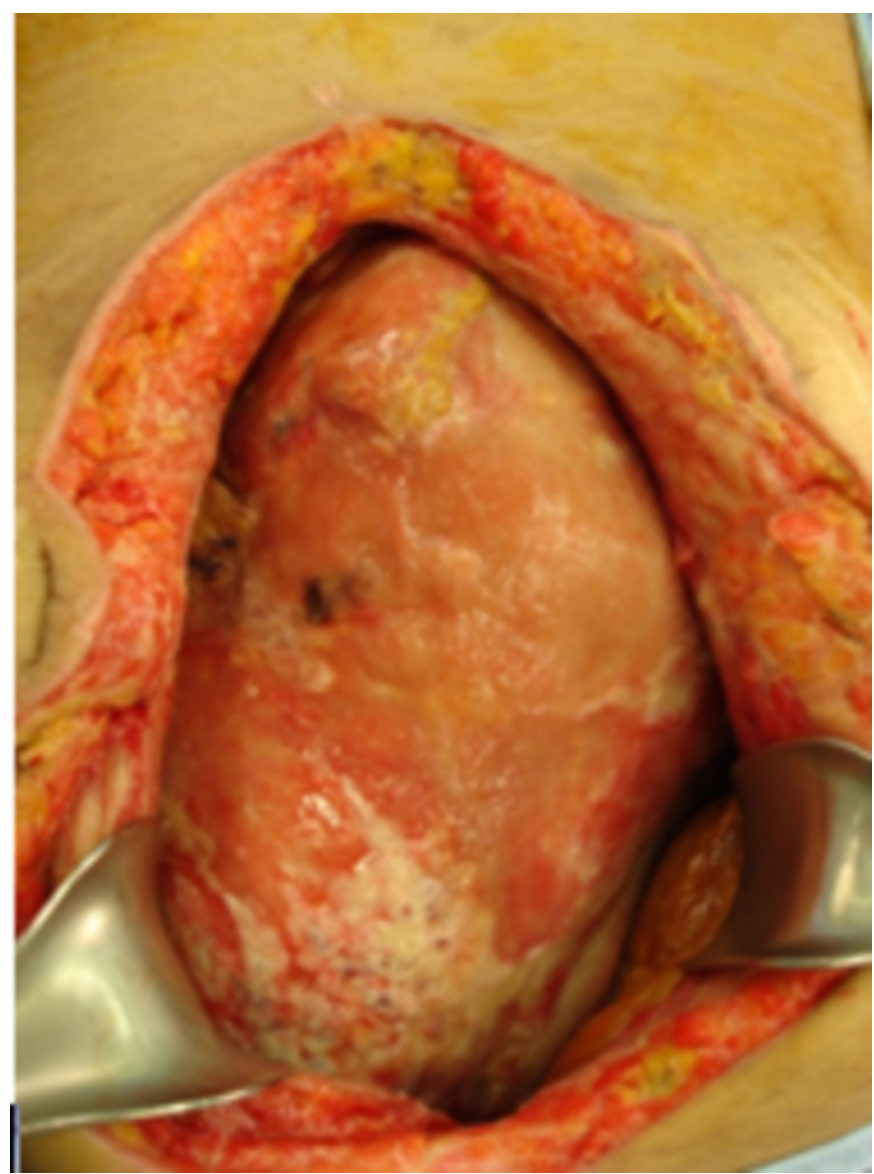

The specimen of the biopsy performed in the operation room confirmed chronic and acute phlogosis, fat necrosis and peritoneal sclerosis. A second culture with susceptibility tests of the peritoneal effusion was required. As a result of these susceptibility tests, the antibiotic therapy was switched to Fluconazole, Amphotericin B, Meropenem and Vancomycin. Despite the 28 days of systemic combined therapy, fever, abdominal pain and distention were persisting (Table 1a). Imaging confirmed the abdominal effusion and encapsulating sclerosis of the peritoneal sac. Cultures were still positive for Candida albicans. 


\section{Figure 2}

Figure 1b. CT scan of the patient's abdomen showing peritoneal sclerosis and parietal abscess.
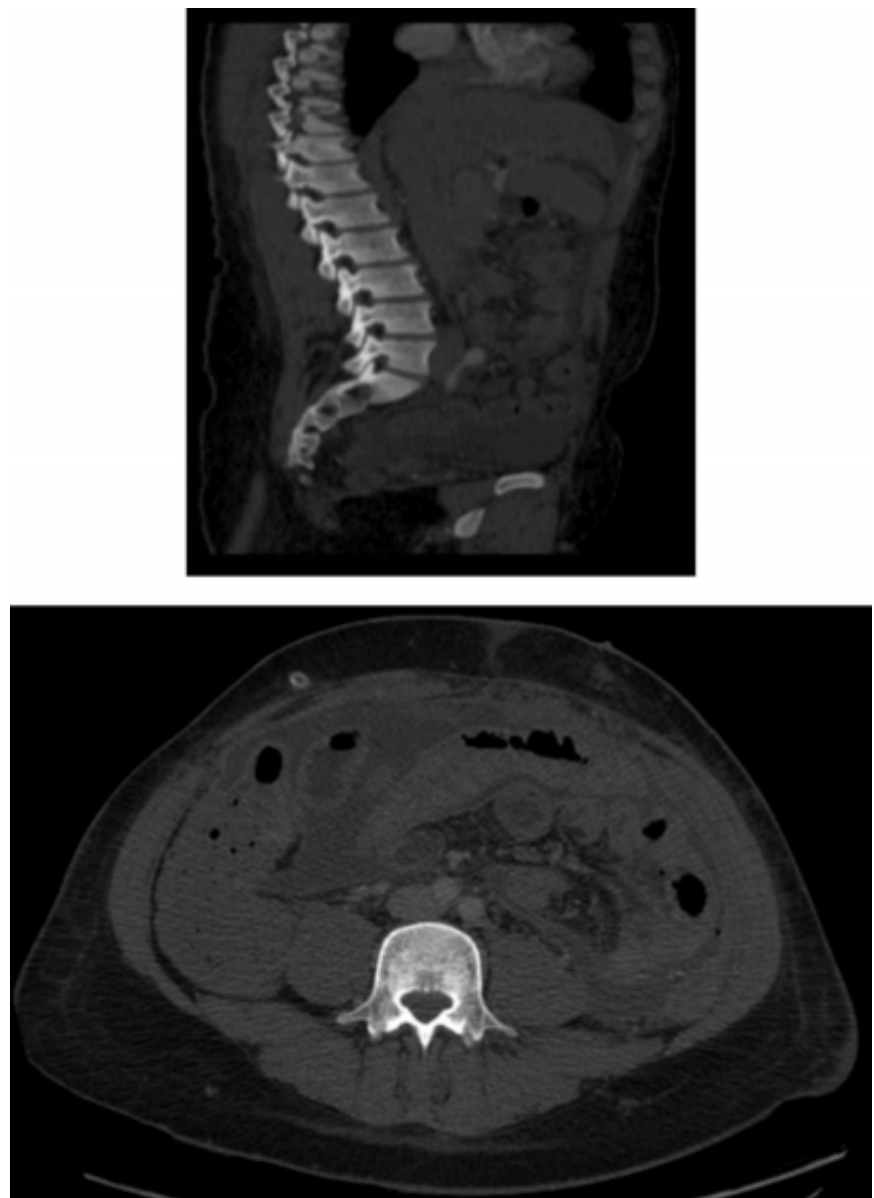

We decided for a second explorative laparotomy with a surgical toilette of the abdominal cavity. We carried out a peritoneal washing with Amphotericin B and saline and a VAWC abdominal system was placed though the laparotomy, over the abdominal viscera and beneath the peritoneum of the abdominal wall with a fenestrated nonadherent plastic and an encapsulated foam dressing. A follow-up schedule was planned which consisted of three applications a week of an intraoperative Amphotericin washing, followed by continued VAWC therapy. At the twelfth cycle of this association, cultures demonstrated the absence of microbiogical growth and the symptoms were resolved: no fever or pain was referred and an appetite improvement was noticed.

\section{Figure 3}

Figure 1c. The fenestrated non-adherent plastic with the encapsulated foam dressing is placed over the abdominal viscera and beneath the peritoneum of the abdominal wall. The negative pressure is applied with a specific drainage tube.

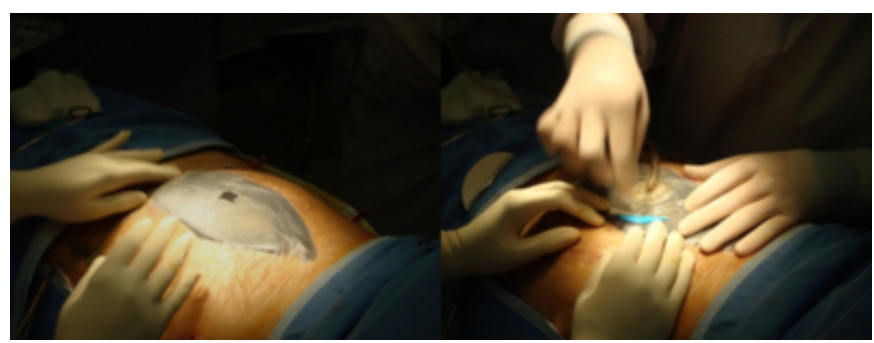

Another three cycles of this scheduled treatment were performed and, on the third negative culture of peritoneal fluids, we decided to definitely close the laparotomy wound and to remove the VAWC abdominal system, leaving only a drainage for seven days. The patient was discharged in good condition and at a ten-month follow-up he is still in good condition, without evidence of abdominal sickness. He continued with intravenous dialysis.

\section{Figure 4}

Table 1a. Timeline of clinical evolution.

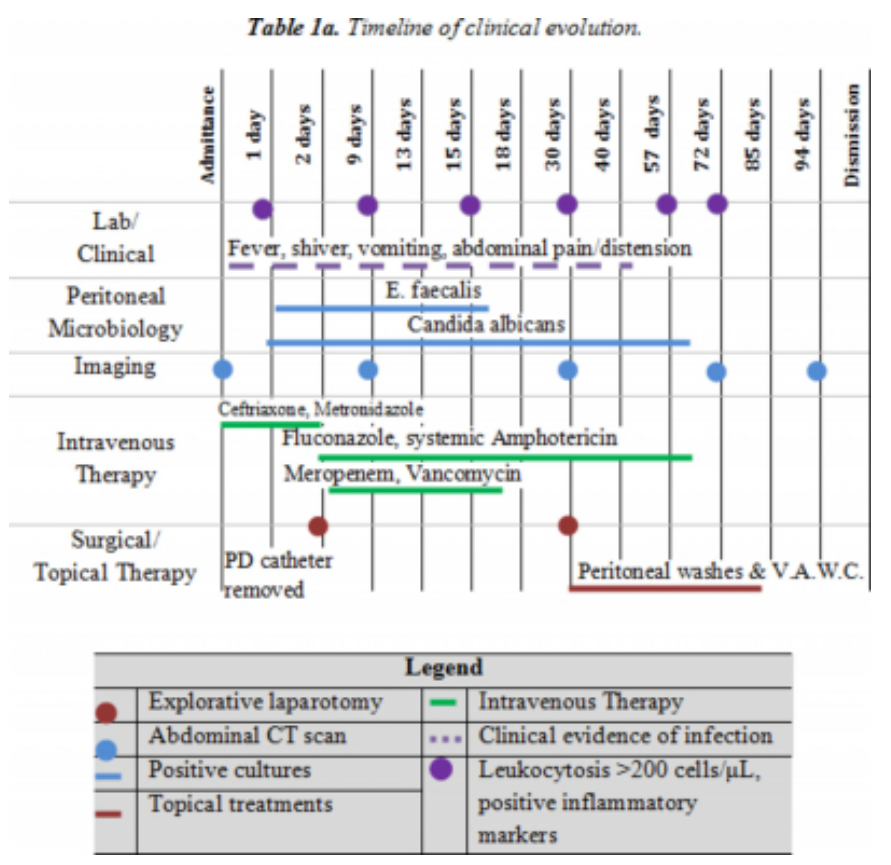

\section{DISCUSSION}

Up to $80 \%$ of all fungal peritonitis episodes in patients on continuous ambulatory peritoneal dialysis (CAPD) are caused by Candida spp. Fungal peritonitis contributes significantly to morbidity, drop-out from the dialysis 
programme and a high level of mortality ${ }^{(1-4)}$. Compared with other causes of peritonitis, fungal peritonitis in $\mathrm{PD}$ is associated with decreased survival ${ }^{(5)}$. Reported mortality rates have ranged from 15 to 45 percent $^{(6)}$. A retrospective review of more than 300,000 patients demonstrated that dialysis patients had an age-adjusted incidence ratio for fungal infections of 9.8 compared to the general population $^{(4)}$.

Major reported risk factors are: recent antibiotic treatment for bacterial peritonitis, invasive diagnostic/therapeutic renal procedures, deferoxamine therapy, immunosuppression for transplantation and comorbidities, such as diabetes or autoimmune disorders $^{(7 ; 8)}$. Nevertheless, a clear relationship between any particular underlying disease and the incidence of PD-related Candida peritonitis has not been shown. When examining the dialysate fluid for a suspected fungal infection, attention should be paid to the duration of the dwell and whether or not peritoneal lavage was performed prior to sample collection; these factors can influence cell counts and culture ${ }^{2}$. With fungal peritonitis, the peritoneal white blood count is almost always greater than 200 cells/ $\square \mathrm{L}$, with a polymorphonuclear cell predominance ${ }^{(3)}$. Candida species usually grow quickly in culture. The diagnosis therefore requires a high level of suspicion, with some cases presenting as culture-negative peritonitis ${ }^{(1)}$.

Reported complications caused by PDCP include sclerosing peritonitis (figure $2 \mathrm{a}$ ), adhesions with resulting bowel obstruction or stricture, invasion of the bowel wall, and abscess formation. Extraperitoneal spread of infection is unusual. The term peritoneal sclerosis (PS) describes one of a vast range of peritoneal alterations, from the manifestations of low clinical impact constantly associated with peritoneal dialysis to the dramatic thickening of the peritoneal membrane, which is rare, but often lifethreatening. It is characterized by a progressive, intraabdominal, inflammatory process resulting in sheets of fibrous tissue that cover, bind, and constrict the viscera ${ }^{(4)}$, thereby compromising the motility and function of the bowel, in $3.3 \%$ of patients under dialysis. Peritoneal sclerosis is a serious, life-threatening complication of CAPD. Most cases had PD duration of more than 4 years ${ }^{(2)}$.

The management of patients with this abdominal catastrophe requires the close cooperation of surgeon, intensivist and microbiologist. Up to now, there have not been any controlled randomized studies demonstrating which treatment regimen has the best outcome in this kind of patient: the peritoneum should therefore be washed until the returning fluid is clear, in order to prevent adhesions and lower the fungal burden. Catheter removal and prompt initiation of antifungal therapy are indicated. Subsequent therapy is based upon culture results, sensitivity, and patient response. In a retrospective review of 70 episodes of fungal peritonitis in a single center, retention of the catheter was associated with increased mortality ${ }^{(5)}$. The choice of an antifungal agent varies based upon the specific infecting organism: fluconazole (200 $\mathrm{mg} / \mathrm{day})$ is recommended for empiric coverage of fungal peritonitis, when there is no suggestion of the identity of the fungus from the inspection of the fluid, and until the cultures have been returned ${ }^{(7)}$.

For patients who have had significant prior exposure to azole antifungals, intravenous amphotericin B $(0.6 \mathrm{mg} / \mathrm{kg}$ per day) or an echinocandin like caspofungin (70 $\mathrm{mg}$ on day one, with subsequent dosing of $50 \mathrm{mg} /$ day) is suggested. After the cultures return, further therapy can be tailored to the specific organism that has been isolated. If the fluid cultures yield a Candida species, such as C. albicans or C. parapsilosis, these are usually susceptible to fluconazole ${ }^{(6)}$.

The duration of a therapy is generally two to four weeks. If the fluid cultures yield an azole-resistant yeast, intravenous amphotericin B for four weeks is recommended. Because nephrotoxicity is not an issue in these patients, these agents likely will only be used in patients who experience severe infusion-related reactions to the deoxycholate formulation ${ }^{(5)}$. The patient should be maintained with haemodialysis during treatment with systemic antifungal agents. Intra-peritoneal instillation of amphotericin B has been attempted and reported to be useful in PDCP. However, abdominal discomfort is a common side effect that has forced interruption of such an approach. Furthermore, some authors have implicated this form of therapy in the development of peritoneal fibrosis ${ }^{(7,8)}$. A short course of intravenous administration of amphotericin B (10 days) has been successful but only when peritoneal catheters were removed $^{(3-6)}$.

Vacuum-assisted wound closure (VAWC), has become the preferred treatment modality for many complex/chronic wounds. The topical negative pressure demonstrated a positive contribution in managing severe peritonitis ${ }^{(9)}$. An open-cell foam (sponge) is inserted in the wound and the sponge is secured into a clear, vapor-permeable and osmotic 
plastic dressing (figure 2b-2c). Tubing extends from the sponge to a disposable collection canister. A portable pump applies $125 \mathrm{mmHg}$ of controlled suction to the system ${ }^{(9)}$. The subatmospheric pressure (suction) is equally distributed across the open wound and evacuates stagnant fluid from the wound. Continual evacuation of stagnant wound fluid promotes local circulation, disposes of cellular waste via the lymphatic system, and decreases tissue edema. Moreover, removal of excess wound fluid reduces the bacterial load of a wound and improves local resistance to infection.

Recently, some studies demonstrated the safety of the VAWC abdominal system. A fenestrated non-adherent plastic with the encapsulated foam dressing must be placed on the abdominal viscera and beneath the peritoneum of the abdominal wall. Amin et al. ${ }^{(9)}$ hypothesizes the possible advantages of using VAWC also in the treatment of multifactorial infective peritonitis. However, at present, no prospective studies have been reported to sustain this theory.

In conclusion, peritoneal sclerosis complicating a Candida infection can negatively affect results of an intravenous antifungal therapy. The strength of this assertion is enhanced by the increased morbidity and mortality associated with this complication. For the treatment of this kind of patients, there is a strong consensus on initial treatment with fluconazole, Amphotericin B and early peritoneal catheter removal ${ }^{(3,5,8,10)}$. Amphotericin B and saline solution intraperitoneal lavages associated with VAWC abdominal system can represent an effective option for common systemic therapy resistant peritonitis. A prospective study on vacuum-assisted wound closure is in progress in our department and this will definitively assess this preliminary data.
Figure 5

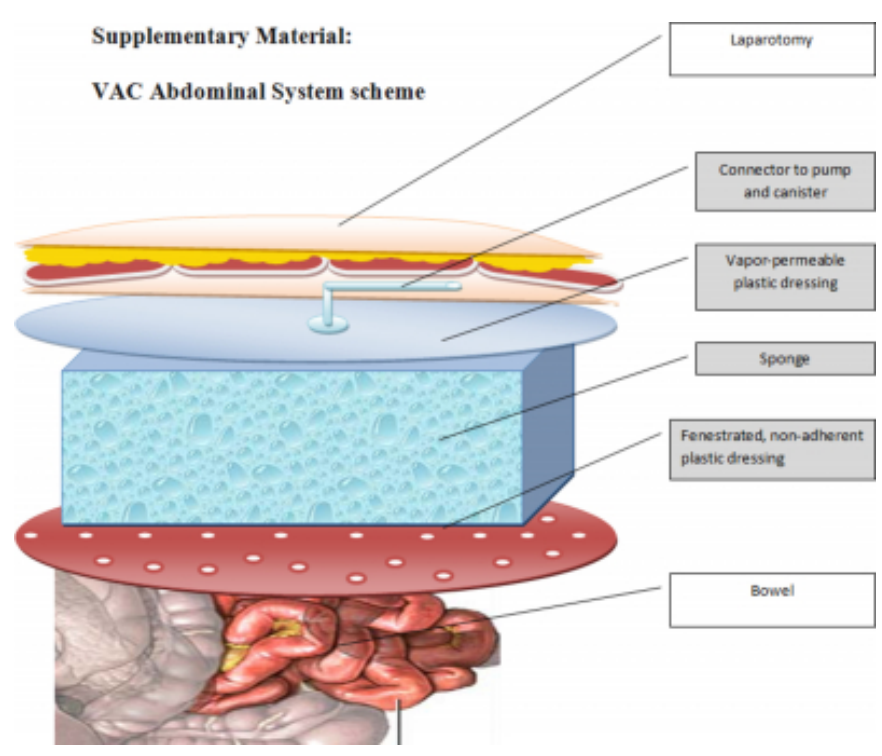

References

1. Michel C, Courdavault L, Al Khayat R, Viron B, Roux P, Mignon F: Fungal peritonitis in patients on peritoneal dialysis. American Journal of Kidney Diseases; 1994; 14: 113-120.

2. Nagappan R, Collins JF, Lee WT: Fungal peritonitis in continuous ambulatory peritoneal dialysis - the Auckland experience. American Journal of Kidney Diseases; 1992; 20 : 492-496.

3. Saran R, Goel S, Khana R: Fungal peritonitis in continuous ambulatory peritoneal dialysis. International Journal of Artificial Organs; 1996; 19: 441-445.

4. Goldie SJ, Kiernan-Troidle L, Torres C, Gorban-Brennan N, Dunne D, Kliger AS, Filkenstein FO: Fungal peritonitis in a large chronic peritoneal dialysis population: a report of 55 episodes. American Journal of Kidney Diseases; 1996; 28: 86-91.

5. Piraino B, Bailie GR, Bernardini J, Boeschoten E, Gupta

A, Holmes C, et al. ISPD Ad Hoc Advisory Committee:

Peritoneal dialysis-related infections recommendations: 2005 update. Perit Dial Int; 2005; 25: 107-31.

6. Blot SI, Vandewoude KH, De Waele JJ: Candida peritonitis. Curr Opin Crit. Care; 2007; 13: 195-9.

7. Abbott KC, Hypolite I, Tveit DJ, et al.: Hospitalizations for fungal infections after initiation of chronic dialysis in the United States. Nephron; 2001; 89: 426.

8. Lien YC, Kuo CC, Liu KL, Hung KY, Huang TM, Huang JW: Encapsulating peritoneal sclerosis. CMAJ; 2009; 181 (3-4): 177

9. Ibrahim A, Shaikh IA: Topical negative pressure in managing severe peritonitis: A positive contribution? World J Gastroenterol; 2009; 15: 3394-3397.

10. Kawaguchi Y: Recommendations of the management of encapsulating peritoneal sclerosis in Japan, 2005: diagnosis, predictive markers, treatment, and preventive measures. Perit Dial Int; 2005; 25(S4): S83-95. 


\section{Author Information}

\section{Buonomo Oreste}

Department of Emergency Surgery, Policlinico Tor Vergata

\section{Balassone Valerio}

Department of Emergency Surgery, Policlinico Tor Vergata

\section{Sarmati Loredana}

Department of Emergency Surgery, Policlinico Tor Vergata

\section{Sena Giuseppina}

Department of Emergency Surgery, Policlinico Tor Vergata

\section{Granai Alessandra Vittoria}

Department of Emergency Surgery, Policlinico Tor Vergata

\section{Ferracci Antonella}

Department of Emergency Surgery, Policlinico Tor Vergata

\section{Ielpo Benedetto}

Department of Emergency Surgery, Policlinico Tor Vergata

\section{Venditti Dario}

Department of Emergency Surgery, Policlinico Tor Vergata

\section{Petrella Giuseppe}

Department of Emergency Surgery, Policlinico Tor Vergata 